\title{
Female stress urinary incontinence: What's old is new again?
}

\author{
Blayne Welk, $M D^{1}$; Kevin V. Carlson, $M D^{2}$ \\ 'Department of Surgery, Western University, London, ON; 2Section of Urology, Department of Surgery, University of Calgary, Calgary, AB; Canada
}

Cite as: Can Urol Assoc J 2017;11 (6Suppl2):S103. htrp://dx.doi.org/10.5489/cuaj.4578

7 he surgical management of female stress urinary incontinence (SUI) has evolved significantly over the last 70 years. The needle suspension procedures of Pereyra (1959), Stamey (1973) and Raz (1981), the autologous fascia pubovaginal sling (popularized in 1978 by Dr. Ed McGuire) and the colposuspensions of Marshall-Marchetti-Kranz (1949) and Burch (1961) persisted for decades after their introduction. The colposuspension and fascial sling are still used today for select patients.

In the 1990s, everything changed. The era of the minimally invasive midurethral sling (MUS) arrived, and this procedure essentially took over as the go-to surgical treatment of SUI. With its rise in popularity, the number of procedures performed and the number of surgeons performing them both increased, suggesting that the gap between the high prevalence of SUI and the number of women who received treatment for it was closing. A strong evidence base documented the efficacy of midurethral slings, and surgical randomized controlled trials, once thought to be fraught with insurmountable challenges, provided level 1 evidence to guide their use.

Things then became a bit more complicated. Concerns about vaginal mesh causing major complications were raised, regulatory warnings were published, and the controversy was escalated by outspoken patients, the media, and legal firms. Neuromodulation and intravesical botulinum toxin for urgency incontinence arrived on the scene and offered new possibilities for treating mixed incontinence. Women with a midurethral sling already in place had recurrent incontinence and the optimal management in this setting was not (and still isn't) known. Finally, mesh complications and the anxiety generated by them drove the creation of an unofficial "meshology" subspecialty. Through all of this, there has been revived interest in other modalities to treat women with SUI who will not accept a mesh implant, those who have failed a prior MUS, and those who have had a complication from one. Indeed, what's old is new again, and a new generation of surgeons is keen to learn techniques that we had only just recently abandoned.

In developing this supplement, we first surveyed the nontrainee Canadian Urological Association (CUA) membership (and received 100 responses). The relevance of this topic is supported by the fact that $80 \%$ of respondents with various fellowship backgrounds see consults for SUI and will perform surgical procedures for stress incontinence when necessary. Not surprisingly, $93 \%$ of these urologists perform midurethral slings, and only a small number of the respondents had fellowship training in incontinence. In urology residency, this has traditionally not been a field in which trainees receive the same amount of operative exposure compared to endourology or uro-oncology. Thus, we felt that this was an "orphan" topic well-deserving of a contemporary review.

The surgical treatment of stress urinary incontinence can be challenging. It takes time to sort out the numerous symptoms which affect the decision to offer surgery. Careful, individualized, and shared decision-making is critical to successful outcomes. The stakes for surgical intervention are high: while successful surgery can dramatically improve a woman's quality of life, complications can have devastating consequences and lead to decisional regret by the patient (and the surgeon). We sincerely thank the CUA for sharing our vision and providing the Continuing Professional Development grant to fund this supplement, and we thank the authors who wrote the included articles.

Competing interests: Dr. Welk has received investigator-initiated funding from Astellas. Dr. Carlson reports no competing personal or financial interests.

Correspondence: Dr. Blayne Welk, Department of Surgery, Western University, London, ON, Canada; bkwelk@gmail.com 